\title{
Follow-Up Study of Growth Hormone Therapy in Children with Kabuki Syndrome: Two-Year Treatment Results
}

\author{
Lieke van Montfort $^{a}$ Willem Jan M. Gerver ${ }^{a}$ Berbel L.S. Kooger ${ }^{a}$ \\ Jogchum Plat $^{b}$ Jörgen Bierauc Connie T.R.M. Stumpel ${ }^{c}$ Dina A. Schott ${ }^{d}$ \\ aDepartment of Paediatrics Endocrinology, Maastricht UMC+, Maastricht, The Netherlands; ${ }^{b}$ Department of Human \\ Biology and Movement Sciences, Maastricht University, Maastricht, The Netherlands; 'Department of Clinical \\ Genetics, Maastricht UMC+, Maastricht, The Netherlands; ${ }^{\mathrm{d} D e p a r t m e n t}$ of Paediatrics, Zuyderland Medical Centre, \\ Heerlen, The Netherlands
}

\section{Keywords \\ Kabuki syndrome · Growth hormone · Cardiovascular risk factors}

\begin{abstract}
Introduction: Kabuki syndrome (KS) is a genetic disorder with characteristic facial dysmorphisms, short stature, hypertension, and obesity later in life. The aim of this study was to evaluate catch-up growth and cardiovascular markers before and during growth hormone (rhGH) treatment in KS children. Methods: This prospective study included 18 children whose KS was genetically established. Each KS subject received rhGH for a period of 2 years. Several measurements were performed before and during treatment: anthropometry, glucose metabolism, lipid profile, markers for endothelial function, and low-grade inflammation. Results: This study found an increase in delta height standard deviation score (SDS) for the whole group of 1.1 SDS after 2 years of rhGH treatment. Baseline metabolic profiles showed no cardiometabolic abnormalities in these children. Although 4 out of 18 children were obese, there were no signs of the metabolic syndrome. During rhGH treatment, serum lowdensity lipoprotein cholesterol concentrations decreased
\end{abstract}

karger@karger.com www.karger.com/hrp

Karger"

GOPEN ACCESS
C 2021 The Author(s)

Published by S. Karger AG, Basel

This is an Open Access article licensed under the Creative Commons Attribution-NonCommercial-4.0 International License (CC BY-NC) (http://www.karger.com/Services/OpenAccessLicense), applicable to the online version of the article only. Usage and distribution for commercial purposes requires written permission. significantly (2.16-1.91 mmol/L, $p=0.04)$. Apolipoprotein B100 concentrations also showed a reduction after 24 months of treatment, but the other lipid and (apo)lipoprotein parameters did not change. While other endothelial function markers were stable, only vascular cell-adhesion molecule- 1 concentrations increased $(1,084-1,161 \mathrm{pg} / \mathrm{mL}$, $p<0.01)$ during rhGH therapy. Furthermore, $\mathrm{BMI}$ and waist circumference improved during treatment. There were no signs of hypertension. Conclusions: At baseline and during rhGH therapy, there were no signs of the metabolic syndrome. This is the first study demonstrating that rhGH treatment in KS children is a safe and effective therapy and that it positively influences linear height without exerting adverse effects on a wide array of cardiovascular risk markers.

(c) 2021 The Author(s)
Published by S. Karger AG, Basel

\section{Introduction}

Kabuki syndrome (KS) is a rare genetic syndrome that is characterized by an unusual facial appearance, postnatal growth retardation, intellectual disability, and various other congenital anomalies $[1,2]$. In 2010, exome sequencing revealed that heterozygous mutations in 
KMT2D (also known as MLL2 gene, OMIM 602113) as a major cause of KS, explaining up to $75 \%$ of the cases [3, 4]. Additionally, mutations in KDM6A (OMIM, 159555) were discovered in $9-13 \%$ of the KS patients [5].

KS children are generally born with a normal birth weight and length. The short stature characterizing KS is mainly caused by postnatal growth delay. The origin for this growth delay is unknown; however, there are some cases of growth hormone (rhGH) deficiency reported in the literature [6-9]. Besides the previously noted features, patients may become very obese toward adolescence without endocrinological explanation for this phenomenon $[10,11]$. Based on this observation, which is in line with the expert opinion of the clinicians of the Kabuki Expertise Centre in Maastricht University Medical Centre, we hypothesized that KS patients are at risk for developing metabolic syndrome.

The literature indicates positive long-term results of rhGH treatment on linear growth and improvement on metabolic risk markers for children with Prader-Willi syndrome $[12,13]$. Moreover, several studies concerning adults treated with rhGH because of rhGH deficiency show beneficial effects on the metabolic outcome $[14,15]$. To date, the effect of rhGH therapy in KS has been investigated in case studies involving rhGH-deficient KS children, but these studies were done without the benefit of understanding the genetic basis of KS $[7-9,16]$. To address this deficiency, a study was conducted at Maastricht University Medical Centre on a cohort of 18 children with genetically proven KS. We recently published the 1 -year treatment results of rhGH therapy on anthropometric variables in this study group [17]. The aims of this study were (1) to evaluate possible aberrations in anthropometric variables, biochemical parameters, and cardiovascular markers in KS children at baseline; (2) to evaluate anthropometric variables after 2 years of treatment with rhGH and after discontinuation of treatment; and (3) to evaluate whether signs of the metabolic syndrome, low-grade inflammation, and endothelial dysfunction are present before the manifestation of clinical symptoms.

\section{Subjects and Methods}

\section{Subjects}

This study was performed as an open-label, prospective, nonrandomized study of KS children (trial registration: NTR4722) at Maastricht University Medical Centre in Maastricht, the Netherlands. Participants were recruited between 2012 and 2014 after informed consent was obtained from their parents. Subjects with a genetically confirmed diagnosis of KS (KMT2D or
KDM6A gene mutation) and prepubertal status were included in the study.

Subjects with diabetes mellitus, an extremely low caloric intake, a previous or active malignancy, rhGH therapy, and children with signs of puberty were excluded. The Medical Ethics Committee of Maastricht University Medical Centre provided ethical approval of the study.

As previously indicated, an arginine and clonidine rhGH-stimulation test was performed in all subjects at the start of the study to rule out rhGH deficiency. The stimulation tests were performed after overnight fasting according to the standard procedures. Most subjects underwent $2 \mathrm{GH}$ stimulation tests on 2 different days: clonidine (Catapresan; Boehringer Ingelheim, Germany) $0.15 \mathrm{mg} /$ $\mathrm{m}^{2}$ given orally and aginine (L-arginine hydrochloride $10 \%$ solution) $0.5 \mathrm{~g} / \mathrm{kg}$ given intravenously over $30 \mathrm{~min}$ at $0 \mathrm{~min}$. Samples for IGF-I, IGFBP-3, and GH determination were obtained at baseline. Samples for GH assessment were also taken at 30, 45, 60, 90, and $120 \mathrm{~min}$ after the administration of arginine and clonidine at $30,60,90,120$, and $150 \mathrm{~min}$. If the maximum GH concentration of both tests was below the $7 \mu \mathrm{g} / \mathrm{L}$, the patient was diagnosed with GHD [18]. Subsequently, rhGH therapy with biosynthetic human rhGH (Genotropin ${ }^{\circledR}$, Pfizer, New York, NY, USA) was initiated. It was administered daily just before bedtime, and the average dose given in our study was $1.0 \mathrm{mg} / \mathrm{m}^{2} /$ day (equivalent to $0.035 \mathrm{mg} / \mathrm{kg}$ / day) for a duration of 24 months. KS children with a GH deficiency received a lower dose of $\mathrm{rhGH}\left(0.7 \mathrm{mg} / \mathrm{m}^{2} /\right.$ day $)$.

\section{Auxological Measurements}

Upon entering the study and at 3-month intervals during the 2 years of treatment, all subjects underwent assessment of height, weight, and waist circumference (WC) and an assessment of the pubertal stages according to Tanner (D.A.S.) [19]. Additionally, anthropometric data were collected for a subgroup of 13 participants 1 year after rhGH therapy was stopped. Measurements were performed according to internationally accepted methods [20,21]. Height, weight, and BMI were expressed in standard deviation scores (SDS), using the reference values of the Netherlands (TNO The Netherlands 2010, with the 2001-2013 Growth Analyser BV, Rotterdam, the Netherlands).

The WC was measured at the part of the trunk located midway between the lower costal margin (bottom of the lower rib) and the iliac crest (top of the pelvic bone) with the person in a standing position. The circumference was measured to the nearest $0.5 \mathrm{~cm}$ at the end of a normal expiration. The waist-to-height ratio (WHtR) was also calculated as a screening tool for the prediction of cardiovascular disease.

The brachial systolic and diastolic blood pressures were determined 3 times in the sitting position using a cuff size corresponding to arm size. An automated device (Dinamap, Pro 300, GE Healthcare, UK) was employed for these readings. Systolic and diastolic blood pressures were expressed in percentiles referencing age- and sex-specific values [22].

Furthermore, the bone age was determined upon the start of the study and at 12 and 24 months. The latter was estimated for all children by the same pediatrician-endocrinologist (D.A.S.) using the Greulich and Pyle atlas method [23].

\section{Biochemical Measurements}

Fasting blood samples were collected before the start of rhGH treatment and at 6-month intervals during the therapy for analyz- 
Table 1. Baseline data (mean \pm SDS) and the auxological data after 1 year and 2 years of rhGH treatment of all 18 subjects with KS

\begin{tabular}{llll}
\hline & Pretreatment & One-year treatment & Two-year treatment \\
\hline Chronological age & $6.86 \pm 2.07$ & $7.97 \pm 2.13$ & $9.00 \pm 2.16$ \\
Bone age & $5.90 \pm 2.14$ & $7.28 \pm 2.37$ & $8.52 \pm 2.46$ \\
HSDS & $-2.40 \pm 1.88$ & $-1.69 \pm 1.94^{*}$ & $-1.30 \pm 1.88^{*}$ \\
Height velocity, cm/year & $5.96 \pm 2.09$ & $9.01 \pm 2.03^{*}$ & $8.19 \pm 1.65^{*}$ \\
Height velocity SDS & $0.41 \pm 2.19$ & $2.78 \pm 1.55^{*}$ & $2.54 \pm 1.46^{*}$ \\
Parent-adjusted HSDS & $-2.07 \pm 1.66$ & $-1.35 \pm 1.61^{*}$ & $-0.95 \pm 1.53^{*}$ \\
Weight SDS & $-1.34 \pm 2.88$ & $-1.16 \pm 2.62$ & $-0.74 \pm 2.23^{*}$ \\
BMI SDS & $0.56 \pm 1.79$ & $0.19 \pm 1.41$ & $0.18 \pm 1.36$ \\
Waist circumference SDS $(n=15)$ & $0.10 \pm 2.19$ & $-0.12 \pm 1.73$ & $0.05 \pm 1.43$ \\
WHtR $(n=15)$ & $0.50 \pm 0.06$ & $0.47 \pm 0.04$ & $0.47 \pm 0.04$ \\
Sitting HSDS & $-1.90 \pm 1.46$ & $-1.10 \pm 1.62^{*}$ & $-0.60 \pm 1.55^{*}$ \\
SLL/SH ratio SDS & $-1.81 \pm 1.84$ & $-1.45 \pm 1.56$ & $-1.45 \pm 1.49$ \\
SBP percentile & $76 \pm 23$ & $60 \pm 25$ & $65 \pm 26$ \\
DBP percentile & $63 \pm 29$ & $61 \pm 20$ & $49 \pm 25$ \\
IGF-SDS & $-0.70 \pm 1.07$ & $1.41 \pm 0.91^{*}$ & $1.60 \pm 0.98^{*}$
\end{tabular}

SDS, standard deviation score; WHtR, waist-to-height ratio; SH, sitting height; SLL, subischial leg length; SBP, systolic blood pressure; DBP, diastolic blood pressure; KS, Kabuki syndrome; rhGH, growth hormone; HSDS, height SDS. $* p<0.05$.

ing a panel of cardiovascular risk markers. Serum and plasma samples for analyzing IGF-I, glucose metabolism, and lipid profiles were separated after centrifugation $\left(3,000 \mathrm{~g}\right.$ for $15 \mathrm{~min}$ at $\left.4^{\circ} \mathrm{C}\right)$ and stored at $-80^{\circ} \mathrm{C}$ until the assays were performed. Plasma tubes were used for the cardiovascular markers $\left(1,300 \mathrm{~g}\right.$ for $15 \mathrm{~min}$ at $4^{\circ} \mathrm{C}$ within $30 \mathrm{~min}$ after blood sampling). After centrifugation, plasma samples were immediately portioned into aliquots and stored at $-80^{\circ} \mathrm{C}$ until analyses at the end of the intervention study. For the plasma amino acid and dimethylarginine (ADMA) determination, fasting blood samples were collected using lithium-heparin tubes $\left(4,000 \mathrm{rpm}, 10 \mathrm{~min}, 4^{\circ} \mathrm{C}\right)$. For ADMA analysis, heparin plasma was deproteinized with $6 \mathrm{mg}$ of solid 5-sulfosalicylic acid (Sigma, St. Louis, MO, USA) per $100 \mu \mathrm{L}$ plasma and was kept frozen at $-80^{\circ} \mathrm{C}$ until analysis.

As previously described, serum IGF-I was measured in venous blood samples with the chemiluminiscence method in an IDSiSYS Multi-Discipline Automated System by Immunodiagnostic Systems Holdings, UK [18]. Fasting plasma glucose concentrations were measured in venous blood samples with the enzymatic hexokinase method using a Roche cobas 8000 modular analyzer (Roche Diagnostics, Rotkreuz, Switzerland). Serum insulin was quantified on the Immulite 2000 XPi Immunoassay System (Siemens Healthcare Diagnostics, Munich, Germany). Homeostatic model assessment of insulin resistance (HOMA-IR) was calculated using the HOMA-IR formula $=(($ fasting insulin $\times$ fasting glucose)/22.5) [24]. A HOMA-IR value $>3$ was chosen as an indicator of reduced insulin sensitivity. Plasma $\mathrm{HbAlc}$ was determined by ion-exchange high-performance liquid chromatography (VARIANT II, Bio-Rad, Hercules, USA).

Serum total cholesterol was measured using the Cholesterol Gen. 2 set as reagents (Roche, Cat. no. 05168538). The color intensity of the formed quinone-imine complex was an indirect photometric measurement of the cholesterol concentration. Serum high-density lipoprotein (HDL) cholesterol concentrations were measured using HDL-Cholesterol plus third generation as reagents. Triglycerides concentrations were measured using standardized triglycerides set (Roche, Cat. no.05171407) as reagents. Low-density lipoprotein (LDL) cholesterol concentrations were calculated using the "Friedewald formula": "total cholesterol" ("triglycerides" $\times 0.453$ ) + "HDL-cholesterol". If the triglyceride concentration was $>4.5 \mathrm{mmol} / \mathrm{L}$ and/or total cholesterol $<1.3$ $\mathrm{mmol} / \mathrm{L}$, the LDL concentration was not calculated but instead measured on an ABX Pentra C400 (HORIBA Medical, Kyoto, Japan).

Apolipoprotein A-I (Apo-A-I)and apolipoprotein B100 (ApoB100) were measured in fasting serum samples using an immunoturbidimetric reaction (HORIBA ABX, Montpellier Cedex, France). Serum Apo-B48 concentrations were measured with a sandwich ELISA kit (Shibayagi) containing a specific anti-Apo-B48 antibody as described previously [25]. Serum Apo$\mathrm{C} 2$ and Apo-C3 were measured with an ELISA kit (Randox). All samples from 1 subject were analyzed within 1 run at the end of the study.

Fasting EDTA-plasma samples were used for measurement of biomarkers for low-grade inflammation (IL-6, IL-8, TNF- $\alpha$, C-reactive protein, serum amyloid $\mathrm{A}$, and endothelial function (soluble vascular cell-adhesion molecule [(VCAM]), soluble intercellular adhesion molecule [ICAM]-1, and soluble endothelial selectin [sEselectin and sP-selectin]) by using a multiarray detection system based on electrochemiluminescence technology (SECTOR Imager 2400, Meso Scale Discovery). All samples from each subject were analyzed within 1 run at the end of the study

Concentration of ADMA was determined in the plasma using the ultra-performance liquid chromatography separation module coupled to an electrospray ionization tandem mass spectrometry (Quattro Premier, Walters, Etten-Leur, the Netherlands) by the Department of Biomedical Genetics at Maastricht University as described by Waterval et al. [26]. 


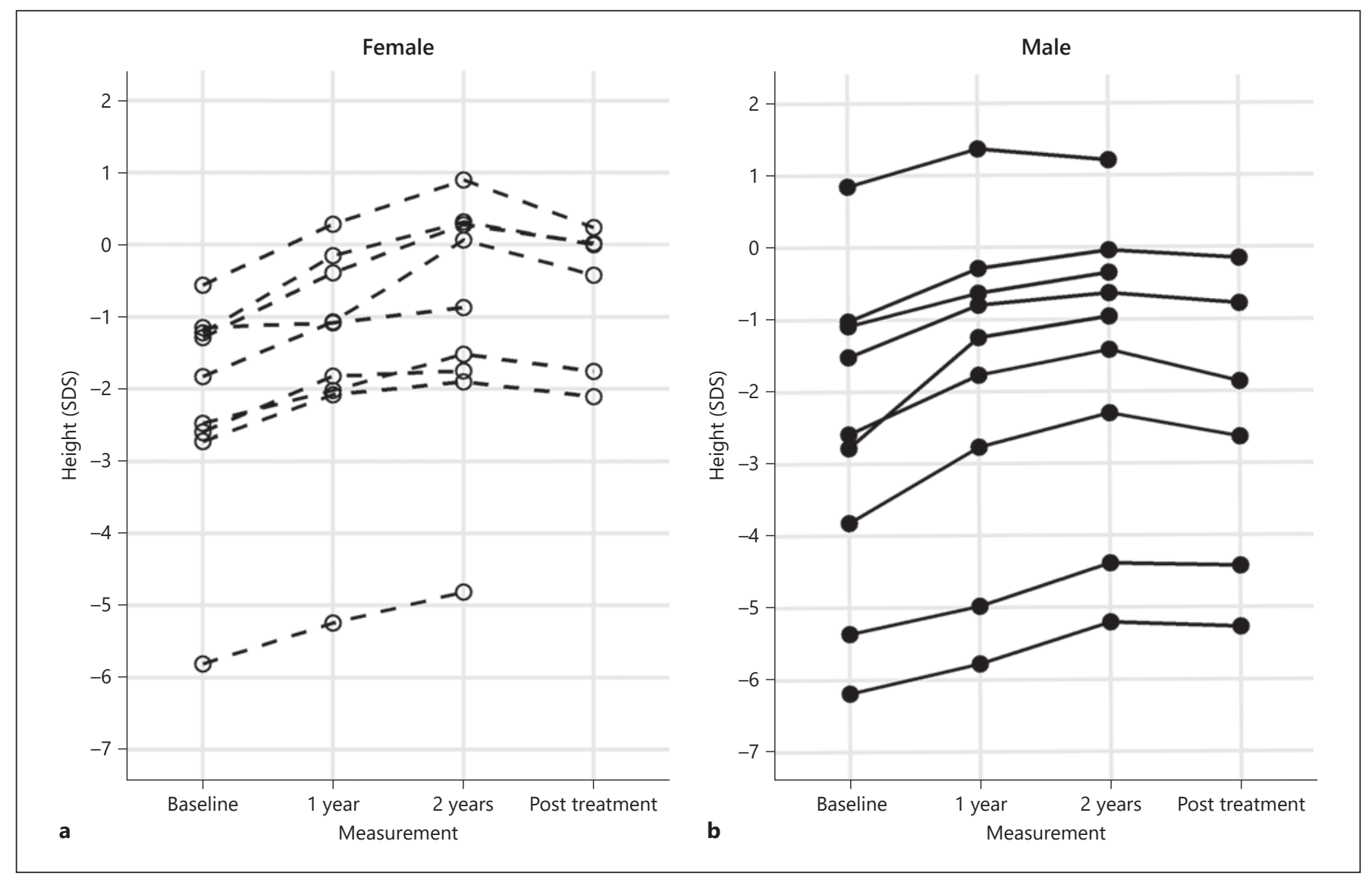

Fig. 1. Height SDS at baseline, after 1 and 2 years of rhGH treatment and 1 year post treatment of all individual 18 subjects with KS. a Female. b Male. KS, Kabuki syndrome; SDS, standard deviation score; rhGH, growth hormone.

Table 2. Auxological data (mean \pm SDS) after 2 years of rhGH treatment and 1 year after cessation rhGH treament in 12 subjects with KS

\begin{tabular}{llllll}
\hline & $\begin{array}{l}\text { Before start } \\
\text { treatment }\end{array}$ & $\begin{array}{l}\text { One-year } \\
\text { treatment }\end{array}$ & $\begin{array}{l}\text { Two-year } \\
\text { treatment }\end{array}$ & $\begin{array}{l}\text { One year after } \\
\text { cessation of treatment }\end{array}$ & $\begin{array}{l}p \text { value } \\
\text { 2-year vs. cessation }\end{array}$ \\
\hline Chronological age & $6.7 \pm 1.99$ & $7.9 \pm 2.07$ & $8.9 \pm 2.08$ & $9.85 \pm 1.90$ & $<0.001$ \\
HSDS & $-2.55 \pm 1.76$ & $-1.82 \pm 1.90$ & $-1.32 \pm 1.91$ & $-1.60 \pm 1.80$ & $<0.001$ \\
Height velocity, cm/year & $6.23 \pm 2.38$ & $9.19 \pm 1.76$ & $8.36 \pm 1.58$ & $4.50 \pm 1.10$ & 0.001 \\
Height velocity SDS & $0.75 \pm 2.45$ & $2.95 \pm 1.58$ & $2.93 \pm 1.46$ & $-0.84 \pm 1.56$ & 0.038 \\
Weight SDS & $-1.74 \pm 2.70$ & $-1.54 \pm 2.76$ & $-1.05 \pm 2.35$ & $-0.74 \pm 2.54$ & 0.026 \\
BMI SDS & $0.14 \pm 1.50$ & $-0.09 \pm 1.35$ & $-0.14 \pm 1.33$ & $0.49 \pm 1.67$ & \\
\hline
\end{tabular}

SDS, standard deviation score; KS, Kabuki syndrome; rhGH, growth hormone; HSDS, height SDS.

\section{Statistical Analysis}

Results for continuous variables were expressed as mean $( \pm$ SDS $)$ and when variables were not normally distributed as median (interquartile range). Binominal data are shown numerically $(n)$. Normal distribution was determined using the KolmogorovSmirnov test. Between-group differences in comparing metabolic risk markers at different time points were, depending on normal distribution, assessed either through Student's $t$ test for paired samples or the Wilcoxon rank test for paired samples. Spearman and Pearson's correlation analyses were used to determine correlations between variables. Furthermore, multiple linear regression analyses were used to investigate the relationship between baseline parameters (e.g., age, height SDS [HSDS], and BMI SDS) and response to treatment by adjusting for multiple variables. All statisti- 


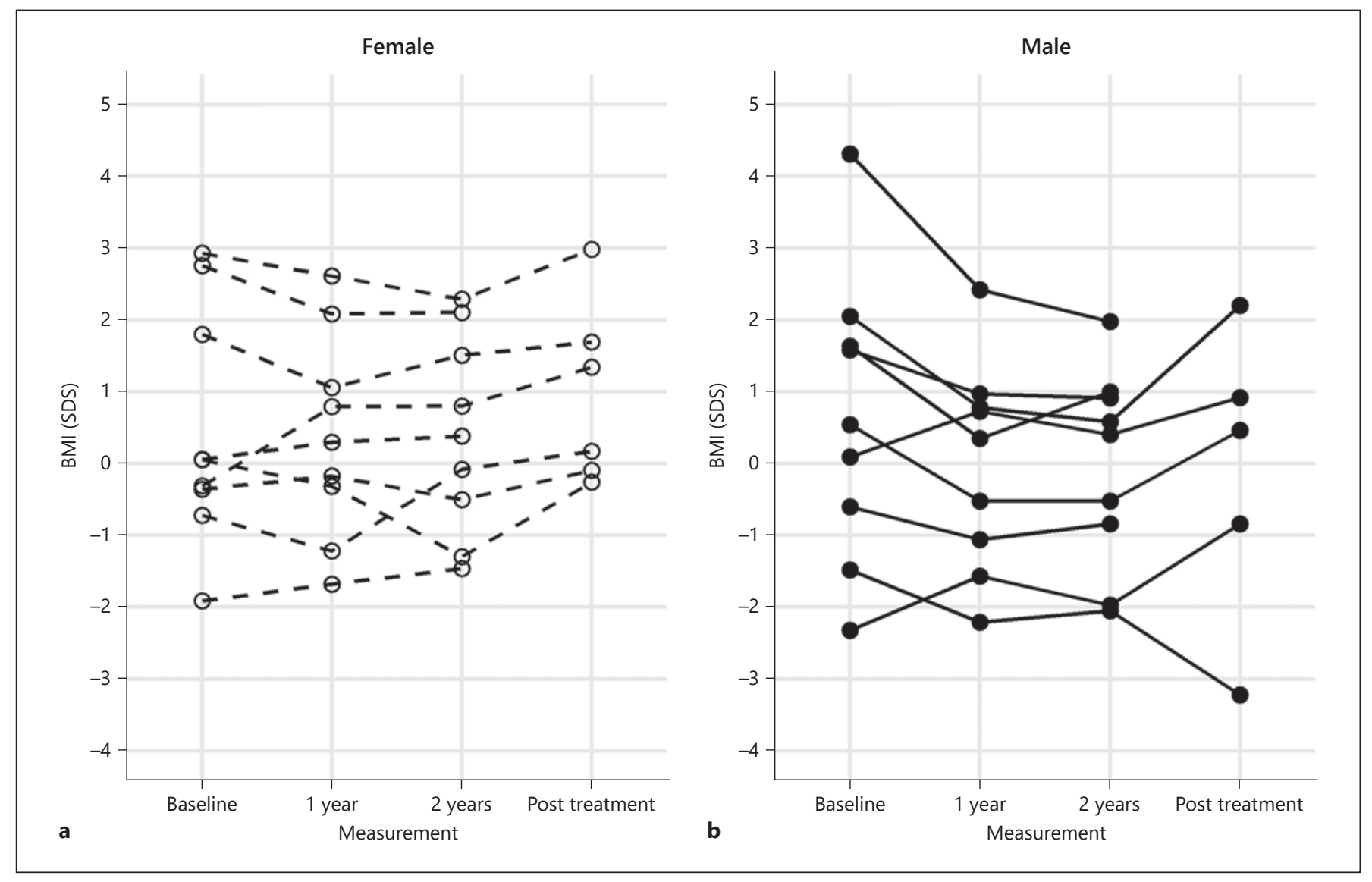

Fig. 2. BMI SDS at baseline, after 1 and 2 years of rhGH treatment and 1 year post treatment of all individual 18 subjects with KS. a Female. b Male. KS, Kabuki syndrome; rhGH, growth hormone.

cal analyses were performed using the software SPSS version 25 (SPSS Inc, Chicago, IL, USA). A $p$ value of $<0.05$ was considered significant.

\section{Results}

The baseline characteristics of the 18 included subjects are provided in Tables 1 and 2. Furthermore, the characteristics of all individual subjects are presented in the online supplementary material (for all online suppl. material, see www.karger.com/doi/10.1159/000519963). In summary, 9 boys and 9 girls were recruited with a mean age of $7.5( \pm 2.3)$ and $6.4( \pm 1.8)$ years, respectively. Fifteen of the KS subjects had a KMT2D gene mutation, and 3 had a KDM6A gene mutation. Based on the results from rhGH stimulation tests, 4 patients were rhGH-deficient. For those patients, additional imaging of the hypothalamic-pituitary region was performed in the patient's region- al hospital, and they were all normal. All patients remained prepubertal during the study period. Thus, sex hormones did not influence the parameters. At baseline, no significant differences were identified between the boys and girls. rhGH treatment was, as mentioned above, initiated with a "standard" rhGH dose. The initial dose of rhGH was altered based on IGF-I levels and/or rhGH response. During 24 months of treatment, the mean dose was $0.96 \pm 0.22 \mathrm{mg} / \mathrm{m}^{2} /$ day $(0.036 \pm 0.010 \mathrm{mg} / \mathrm{kg} /$ day $)$.

\section{Anthropometry}

The anthropometric measurements at baseline and after 1 and 2 years of rhGH treatment are displayed in Table 1 and Figures 1 and 2. Additionally, data of individual subjects are provided in the online supplementary table. HSDS improved from $-2.40( \pm 1.88)$ at baseline to $-1.30( \pm 1.88)$ after 2 years of treatment. Hence, an increase in $\triangle$ HSDS of 1.1 SDS occurred post-2 years of rhGH substitution. 


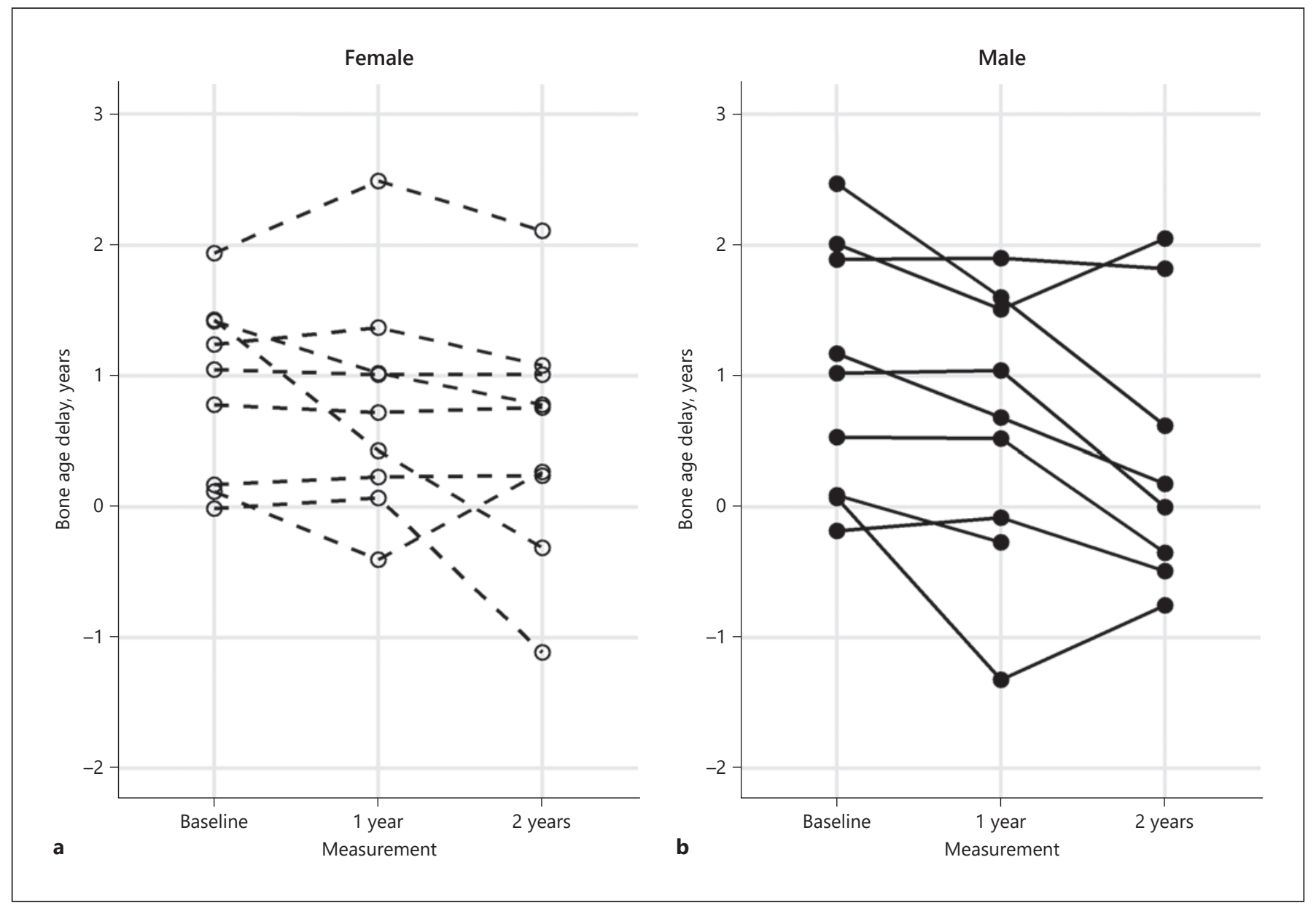

Fig. 3. Bone age delay (years) at baseline and after 1 and 2 years of rhGH treatment of all individual 18 subjects with KS. KS, a Female. b Male. Kabuki syndrome; rhGH, growth hormone.

Individually, responsiveness to rhGH therapy, defined as 0.5 SDS change in height, was identified in 13/18 (72\%) children after 1 year and 16/18 (89\%) children after 2 years of treatment. Furthermore, all 16 subjects exceeded a height gain of 0.5 SDS, by surpassing 0.7 SDS. Two children did experience less growth response with an increase in HSDS of 0.28 and 0.36 , respectively. One of them was suffering from headaches that were initially thought to be a side effect of rhGH therapy, and the rhGH dose was consequently lowered to about $0.017 \mathrm{mg} /$ $\mathrm{kg} /$ day. The other child had already exhibited a normal growth pattern at the start of treatment and was somewhat older than the average age of the study group ( 9.47 years). Analysis of the results by multiple linear regression revealed that a younger age at the start of treatment was associated with a greater $\triangle$ HSDS adjusted for baseline HSDS, baseline BMI, and baseline IGF-I $\{(\mathrm{F}[4,13]$ $=5.15, p=0.010)$, with an $\mathrm{R}^{2}$ of 0.61 . Subjects' predicted $\triangle \mathrm{HSDS}$ is equal to $2.30-0.115$ (age at onset in years) +0.01 (BMI at onset) -0.009 (HSDS at onset) -0.004 (IGF-I SDS at onset). Both baseline IGF-I SDS and age were significant predictors of $\triangle \mathrm{HSDS}(p=0.02$ and $p=$ 0.01 , respectively)\}.

No statistically significant intergroup differences were seen in height gain SDS after the 2-year rhGH therapy for the subgroups KMT2D versus KDM6A or for short stature versus normal stature ( $p=0.44$ and $p=0.77)$. Corresponding numbers for the non-GH-deficiency and GHdeficiency subgroups were 1.02 and $1.42 \Delta \mathrm{HSDS}$, respectively $(p=0.15)$. Nevertheless, all subgroups showed a significant increase in HSDS when compared to baseline. Boys and girls experienced almost the same increase in $\Delta$ HSDS, 1.07 and 1.15, correspondingly.

At the beginning of the study, the mean bone age delay of the subjects was approximately 1 year. After 2 years of rhGH therapy, the mean bone age advanced from 5.90 to 
Table 3. Cardiovascular and metabolic parameters at baseline and after 12 and 24 months of rhGH treatment

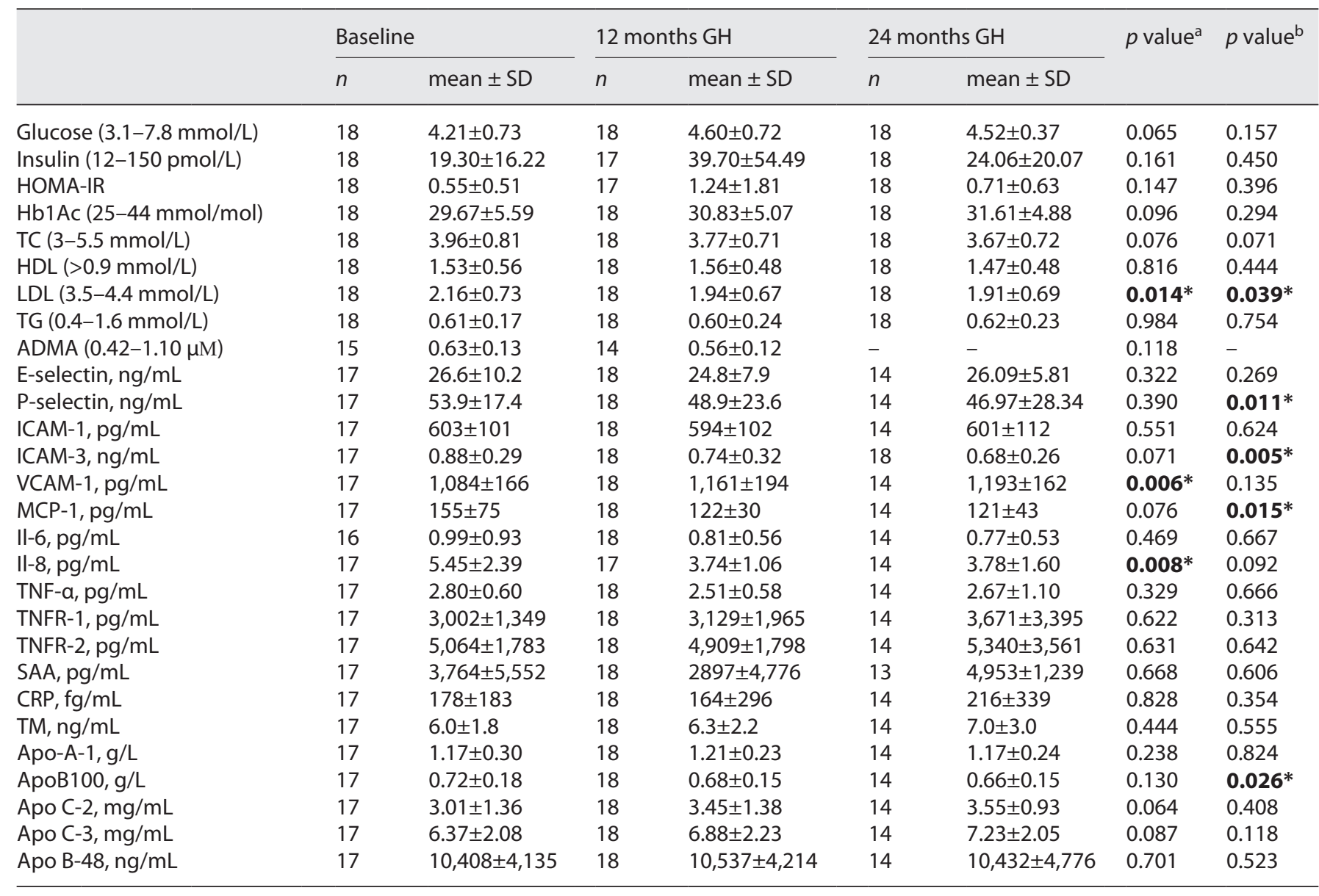

a $p$ value 12 months compared to baseline; ${ }^{b} p$ value 24 months compared to baseline. SDS, standard deviation score; $n$, number; HOMAIR, homeostatic model assessment-insulin resistance; TC, total cholesterol; HDL, high-density lipoprotein; LDL, low-density lipoprotein; TC, triglycerides; ADMA, plasma amino acid and dimethylarginine; ICAM, intercellular adhesion molecule; VCAM, vascular cell-adhesion molecule; MCP, monocyte chemoattractant protein; IL, interleukin; TNF, tumor necrosis factor; TNFR, TNF receptor; SAA, serum amyloid A; CRP, C-reactive protein; TM, thrombomodulin; Apo, apolipoprotein; rhGH, growth hormone.

8.52 years, as is shown in Figure 3. This advance in the bone age resulted in a significant decrease in the difference between BA and CA (chronological age) from approximately 1 year to 6 months. The mean $\triangle \mathrm{BA} / \Delta \mathrm{CA}$ was $1.25 \pm 0.45$ and $1.26 \pm 0.31$ after 1 and 2 years of treatment, respectively.

During treatment, no significant changes in body proportions were measured. A significant difference in the sitting height was observed which was greater than the difference in height. Nevertheless, no significant alteration in the subischial leg length/sitting height ratio was noted. This ratio decreased in the first year of treatment from -1.81 SDS to -1.45 SDS and remained stable during further treatment.

rhGH Treatment in KS Children
Throughout this study, every participant received rhGH treatment for a duration of 24 months. After this period, only 3 participants had an indication for continuing rhGH substitution according to the current guidelines of the Dutch Growth Research Foundation. These guidelines declare that rhGH treatment is indicated when patients (1) suffer from short stature, (2) have a delayed bone age, (3) other causes than rhGH deficiency for growth delay are ruled out, and (4) rhGH deficiency is demonstrated by 2 rhGH stimulation tests. Subsequently, in 15 participants, rhGH treatment was ended after 24 months. Data on linear growth were obtained for 12 participants in this subgroup 1 year after cessation of rhGH treatment. In this subgroup, HSDS declined significantly

Horm Res Paediatr 2021;94:285-296 291 
from -1.32 to $-1.60(p<0.001)$. In addition, a significant decrease in the height velocity was also observed $(p<$ 0.001), which is displayed in Table 2 and Figure 1.

Before initiating rhGH treatment, overall BMI SDS was $0.56 \pm 1.79$, with a mean of 0.64 (boys) and 0.48 (girls). Overall BMI SDS decreased to $0.19 \pm 1.41$ after 1 year of rhGH treatment and remained stable at this level after the second year. However, BMI increased significantly to 0.49 \pm 1.671 year after discontinuation of rhGH therapy, as is demonstrated in Table 2 and Figure 2. At start of the study, 4 patients were obese, defined as BMI $>2$ SDS. Their BMI SDS decreased from 3.01 to 1.74 after 2 years of treatment, representing an ongoing nearly significant effect $(p=0.05)$. The WC and WHtR changed, respectively, from -0.10 to $0.05 \operatorname{SDS}(p=0.44)$ and 0.50 to $0.47(p<0.01)$, respectively. The WHtR ratio decreased from 0.58 to 0.51 in the obese group which is not statistically significant $(p=0.145)$.

\section{Blood Pressure}

Blood pressure values were all within the normal range at baseline, as well as after 1 and 2 years of rhGH therapy. There was no significant difference between boys and girls, and there was no association with BMI and WC.

\section{Glucose Metabolism}

At baseline, fasting glucose, insulin, and $\mathrm{HbAlc}$ concentrations, as well as the HOMA-IR, were all within the normal range. After 2 years of rhGH treatment, there were no significant changes in $\mathrm{HbAlc}$, glucose, insulin, and HOMA-IR. However, there was a trend toward the development of insulin resistance after 12 months (Table 3) since both fasting glucose and insulin concentrations increased slightly. This tendency completely disappeared after 2 years of treatment. In terms of disaggregating effects between genders, only girls presented a significant increase in fasting glucose concentrations after 1 year of rhGH treatment $(p=0.02)$.

\section{Igf-I}

Favorable results were observed for serum IGF-1 levels. These levels significantly increased from a mean of $-0.70 \pm 1.07$ at the start to $1.60 \pm 0.98$ after 24 months of rhGH therapy. Additionally, serum IGF-1 SDS levels $>2$ were seen in 6 patients during treatment. rhGH dosage was adjusted for these patients which resulted in a lowering of serum IGF-I SDS levels below 2 .

\section{Lipid and Lipoprotein Metabolism}

All parameters associated with lipid and lipoprotein metabolism were within normal ranges at baseline and after 12 and 24 months of rhGH therapy. A significant decrease was seen for LDL cholesterol concentrations during the whole study period when compared to baseline (Table 3). This decline was not correlated with the decrease in BMI or the WC. In line with LDL, a statistically significant decrease of ApoB100 concentrations was observed at 24 months. The ApoB100/Apo-A1 ratio, a known predictor for the risk of cardiovascular disease, significantly decreased from 0.66 at baseline to 0.58 after 1 year of treatment $(p=0.03)$. However, after 2 years of treatment, the ApoB100/Apo-A1 ratio increased to 0.61 and therefore no longer represented a significant difference.

\section{Markers for Endothelial Function and Chronic Low-} Grade Inflammation

Endothelial function was evaluated by measuring ADMA, intercellular adhesion molecule-1, VCAM-1, intercellular adhesion molecule-3 (ICAM-3), E and P-selectin, and monocyte chemoattractant protein-1 (MCP1) levels. With the exception of VCAM-1, all of these remained stable during the first year of rhGH treatment. Significant alterations were evident for P-selectin, ICAM3 , and MCP-1 after 2 years. P-selectin levels decreased significantly after 24 months of rhGH treatment. Furthermore, a declining trend in ICAM-3 and MCP-1 levels was already seen at 12 months of therapy and reached significance after 24 months of rhGH therapy.

The presence of a low-grade inflammatory state was evaluated by measurements of TNF- $\alpha$, TNF receptors, Il6, Il-8, C-reactive protein, and serum amyloid A concentrations. With one exception, no changes in these parameters were found before and after 12- and 24-month rhGH treatments. The chemokine Il-8 initially decreased significantly; however, this effect was no longer perceived after the 2-year rhGH treatment.

\section{Discussion}

In addition to the well-known congenital anomalies such as distinctive facial features, postnatal growth retardation is one of the main clinical characteristic of children with KS. This study provides the first long-term analysis of the effects of rhGH therapy on catch-up growth and cardiovascular risk markers. After 2 years of rhGH treatment, a significant increase on overall linear growth was seen with an increase in the mean height from -2.40 SDS to $-1.30 \mathrm{SD}$ and with a responsiveness to treatment of $89 \%$. In addition to growth promotion, improvements in 
$\mathrm{BMI}$ and WCHt were noticed without signs of cardiovascular abnormalities.

The cause of the short stature in KS remains unclear. Niikawa et al. [6] reported about postnatal growth retardation in male subjects. Their finding was validated in a northern European study on 39 individuals with a genetically proven diagnosis of KS [27]. A genotype-phenotype correlation was not observed. Further research was done on the GH-IGF-I axis in a prospective study on 18 genetically confirmed KS individuals [18]. A combination of both arginine and clonidine rhGH stimulations tests demonstrated a lack of GH response in $27.8 \%$ of the KS individuals. However, IGF-I levels did not correspond to the GH stimulations tests, while none of the GHD KS patients had a low IGF-I level. These results indicate that GHD is not the only cause of short stature in KS patients. Therefore, we conclude that the utility of performing $\mathrm{GH}$-stimulation tests on KS children as an indication of $\mathrm{GH}$ status in short stature is questionable.

Nevertheless, rhGH treatment in KS children produced significant catch-up growth. Furthermore, a significant association was seen between the age of initiating treatment and the growth response. In this study, KS patients received rhGH therapy for a period of 2 years. This exerted a positive effect on linear growth, which partially diminished when rhGH therapy was stopped. One year after the end of rhGH treatment, HSDS deflected to -0.23 . Results about the effect of discontinuing rhGH treatment before achieving final height are scarce. The limited literature about this subject concerns study groups with patients suffering of other syndromes. Nevertheless, these studies report comparable results on linear growth after discontinuation of rhGH treatment. Additionally, they report about metabolic deterioration after cessation of rhGH therapy [28-31]. However, the metabolic effect of discontinuing rhGH was not included in this study, but the decline in linear growth provides another argument that rhGH is an effective treatment in KS children.

Bone age measurements are an important step in diagnosing growth-related diseases in patients with shortstature and are delayed in patients with GHD, SGA, hypothyroidism, malnutrition, and chronic illness [32]. GH and IGF-I are traditionally considered potent stimulators of bone growth. After GH treatment, increased serum IGF-I levels stimulate growth plate development, resulting in BA progression and impact final height. During 2 years of rhGH treatment, our study showed a bone age progression from approximately 1 year to 6 months. Studies with similar conditions to ours (prepubertal at start of rhGH treatment, same dosing, and 2 years of

rhGH Treatment in KS Children treatment) by Cohen et al. [33] and Kang et al. [34] in GHD children reported 1.2 and 1.28 years of BA progression/year of CA respectively, which were consistent with our findings (1.25 of BA progression/year of CA). Our results suggest that the 2-year $\mathrm{GH}$ treatment benefits on HSDS for CA are most likely not to affect the final height gain.

During the first year of rhGH treatment in our study, the increase in sitting height was slightly greater than the increase in height. However, this did not result in a significant alteration in the subischial leg length/sitting height ratio. Furthermore, this ratio did not change at all during the second year of rhGH treatment, thereby representing normal body proportions during rhGH therapy.

To our knowledge, no past research reported on metabolic markers in KS patients, and little is known about the phenotype of adult KS patients. However, KS children tend toward obesity during adolescence $[10,11]$. Furthermore, fat distribution is described as mainly truncal in KS [11]. Therefore, we hypothesized that KS patients are predisposed to an elevated risk for developing metabolic syndrome in adulthood. While our study population was prepubertal, only 4 patients were suffering from obesity. Nevertheless, we found a reduction in BMI after 2 years of rhGH treatment from 0.56 SDS to 0.18 SDS in the total study population, with a nearly significant $(p=0.05)$ decrease in the obesity subgroup $(n=4)$. Additionally, there was a significant correlation between the BMI, WC, and the WHtR in the total study population, confirming that the fat distribution in our population was truncal. Studies demonstrated that rhGH treatment induced reductions in visceral adipose tissue and improved insulin sensitivity $[35,36]$. Indeed, there was a reduction in the WC and WHtR on rhGH in our KS children population. Despite the small study size, this indication is clinically important with regard to the prevention of truncal obesity and therefore the eventual risk on the development of the metabolic syndrome. Next to obesity, hypertension and dyslipidemia are typical components of the metabolic syndrome as defined by elevated serum total and LDL cholesterol and/or triglycerides and decreased HDL cholesterol concentrations [37]. Since there is, at best, scant understanding about the MS or cardiovascular complications in KS subjects, blood pressure and cardiovascular parameters were also studied. At baseline and after 24 months of rhGH therapy, blood pressure remained normal, and there were no significant correlations with BMI or the WC. The same was true for dyslipidemia. Notably, the LDL cholesterol concentrations significantly decreased during rhGH treatment. Since ApoB100 concen- 
trations were also lowered, it can be suggested that not only LDL cholesterol concentrations but also the number of LDL particles was reduced. A recent meta-analysis by Giagulli et al. [38] revealed an overall reduction in LDL levels in GHD patients, which echoes the findings in our study. This finding points to a preventative intervention against cardiovascular problems later in life.

In children, it is known that high IGF-I levels and treatment with rhGH are associated with increased insulin levels and correlate with growth response in some cases $[39,40]$. In this study, the IGF-I levels significantly increased during rhGH therapy. Fasting glucose, insulin, HbAlc concentrations, and the HOMA-IR were all within the normal range at baseline and remained normal after 2 years of rhGH treatment despite the association between rhGH administration and insulin resistance.

$\mathrm{RhGH}$ deficiency in adults is associated with an increase in visceral adiposity, adverse serum lipid profiles, vascular endothelial dysfunction, and reduced exercise capacity [41-43]. It is known that obesity is accompanied by chronic inflammation of adipose tissue. While data suggest that GH plays an inhibitory role on TNF- $\alpha$ release, current studies are inconclusive [44]. However, PWS positive associations were found between TNF- $\alpha$ and body weight. Furthermore, rhGH treatment decreased the levels of the inflammatory markers, albeit not to a statistically significant level [45]. We observed a similar trend with the reduction of TNF- $\alpha$ levels in the first year, and only after 2 years of treatment, this disappeared. The same was true for IL-8.

ADMA is a competitive inhibitor of nitric oxide synthase, which is responsible for most vascular nitric oxide production. A high prevalence of elevated plasma ADMA is observed in patients with hypercholesterolemia, hypertension, renal failure, diabetes mellitus, cardiovascular disease, and many other clinical disorders, even among apparently healthy persons. Andrade et al. [46] studied ADMA levels in healthy children and made a biochemical profile for ADMA levels with a mean of $0.61 \mu \mathrm{M}(0.42-$ 1.10). Our study group had the same mean level at baseline, and during rhGH treatment, the levels even exhibited a slight decline. Therefore, we can conclude that our population of KS children has normal ADMA levels and does not seem to have any signs of cardiovascular problems.

Studies of endothelial function in GHD adults using biochemical methods revealed that 12 months of rhGH treatment led to a decrease of these biochemical parameters similar to that experienced by healthy controls. In the literature, a strong correlation exists between the observed changes in IGF-1 and these parameters [47]. In patients with the acromegaly serum, ICAM and VCAM levels are significantly higher than those in the control group [48]. Also, in our group, the VCAM-1 concentration was significantly elevated during the first year of treatment. The other parameters remained stable, although ICAM-3 concentrations exhibited a significant tendency toward lower values. In conclusion, we can say that there are inconsistent outcomes regarding endothelial function when employing biochemical methods in KS children during rhGH replacement.

In conclusion, we demonstrated that there was a convincing positive effect of rhGH treatment on linear growth in KS children. Furthermore, there were no severe cardiometabolic abnormalities in KS children at baseline or during rhGH treatment. Moreover, serum LDL cholesterol and ApoB100 concentrations were lowered. Biomarkers for endothelial function and inflammation did not change during therapy. Additionally, no important side effects were reported. Therefore, the results from this study demonstrate that rhGH treatment in KS children is a safe and effective means of promoting catch-up growth during a 2-year therapeutic approach. Long-term followup will be mandatory to evaluate the effect of rhGH on final height, the tendency toward obesity during adolescence, and the eventual risk on metabolic syndrome.

\section{Acknowledgments}

We are grateful to the Dutch Kabuki Syndrome Network and to the individuals with KS and their families/caregivers for their willingness to participate in this study.

\section{Statement of Ethics}

This study was performed as an open-label, prospective, nonrandomized study of KS children (trial registration: NTR4722, study approval reference number: 2012-000432-26) at Maastricht University. The Medical Ethics Committee of the Maastricht University Medical Centre provided ethical approval of the study. Written informed consent was obtained from all parents.

\section{Conflict of Interest Statement}

All authors report no conflicts of interest to declare.

\section{Funding Sources}

The study was financially supported by Pfizer. The funder was not involved in the study design, collection, analysis and interpretation of data, writing of the report, or the decision to submit the manuscript for publication. 


\section{Author Contributions}

Drs. Lieke van Monfort was responsible for data analysis and writing the manuscript. Dr. Willem-Jan Gerver contributed to the acquisition and design of the study as well as both critically revising the article in terms of important intellectual content and in providing final approval of the version to be submitted. Drs. Berbel Kooger preformed analysis of biomarker data. Prof. Jogchum Plat contributed to the acquisition, analysis, and interpretation of cardiovascular biomarker data and in critically revising the article in terms of important intellectual content. Dr. Jorgen Bierau contributed to the acquisition and analysis of the ADMA data.
Prof. Constance Stumpel was responsible for the genetic content of the final version to be submitted. Dr. Dina Schott was responsible for study design, data curation, literature review, supervision, drafting and critically revising the article, as well as providing final approval of the version to be submitted.

\section{Data Availability Statement}

The data that support the findings of this study are available on request from the corresponding author.

\section{References}

1 Kuroki Y, Suzuki Y, Chyo H, Hata A, Matsui I. A new malformation syndrome of long palpebral fissures, large ears, depressed nasal tip, and skeletal anomalies associated with postnatal dwarfism and mental retardation. J Pediatr. 1981 Oct;99(4):570-3.

2 Niikawa N, Matsuura N, Fukushima Y, Ohsawa T, Kajii T. Kabuki make-up syndrome: a syndrome of mental retardation, unusual facies, large and protruding ears, and postnatal growth deficiency. J Pediatr. 1981 Oct;99(4): $565-9$.

$3 \mathrm{Ng} \mathrm{SB}$, Bigham AW, Buckingham KJ, Hannibal MC, McMillin MJ, Gildersleeve HI, et al. Exome sequencing identifies MLL2 mutations as a cause of Kabuki syndrome. Nat Genet. 2010 Sep;42(9):790-3.

4 Paulussen AD, Stegmann AP, Blok MJ, Tserpelis D, Posma-Velter C, Detisch Y, et al. MLL2 mutation spectrum in 45 patients with Kabuki syndrome. Hum Mutat. 2011 Feb; 32(2):E2018-25.

5 Miyake N, Mizuno S, Okamoto N, Ohashi H, Shiina M, Ogata K, et al. KDM6A point mutations cause Kabuki syndrome. Hum Mutat. 2013 Jan;34(1):108-10.

6 Niikawa N, Kuroki Y, Kajii T, Matsuura N, Ishikiriyama $\mathrm{S}$, Tonoki $\mathrm{H}$, et al. Kabuki makeup (Niikawa-Kuroki) syndrome: a study of 62 patients. Am J Med Genet. 1988 Nov;31(3): 565-89.

7 Tawa R, Kaino Y, Ito T, Goto Y, Kida K, Matsuda $\mathrm{H}$. A case of Kabuki make-up syndrome with central diabetes insipidus and growth hormone neurosecretory dysfunction. Acta Paediatr Jpn. 1994 Aug;36(4):412-5.

8 Devriendt K, Lemli L, Craen M, de Zegher F. Growth hormone deficiency and premature thelarche in a female infant with kabuki makeup syndrome. Horm Res. 1995;43(6): 303-6.

9 Gabrielli O, Bruni S, Bruschi B, Carloni I, Coppa GV. Kabuki syndrome and growth hormone deficiency: description of a case treated by long-term hormone replacement. Clin Dysmorphol. 2002 Jan;11(1):71-2.

10 White SM, Thompson EM, Kidd A, Savarirayan R, Turner A, Amor D, et al. Growth, behavior, and clinical findings in 27 patients with Kabuki (Niikawa-Kuroki) syndrome. Am J Med Genet A. 2004 Jun 1;127A(2):11827.

11 Schrander-Stumpel CT, Spruyt L, Curfs LM, Defloor T, Schrander JJ. Kabuki syndrome: clinical data in 20 patients, literature review, and further guidelines for preventive management. Am J Med Genet A. 2005 Jan 30; $132 \mathrm{~A}(3): 234-43$.

12 van Mil EG, Westerterp KR, Gerver WJ, Van Marken Lichtenbelt WD, Kester AD, Saris WH. Body composition in prader-willi syndrome compared with nonsyndromal obesity: relationship to physical activity and growth hormone function. J Pediatr. 2001 Nov; 139(5):708-14.

13 de Lind van Wijngaarden RF, Cianflone K, Gao Y, Leunissen RW, Hokken-Koelega AC. Cardiovascular and metabolic risk profile and acylation-stimulating protein levels in children with Prader-Willi syndrome and effects of growth hormone treatment. J Clin Endocrinol Metab. 2010 Apr;95(4):1758-66.

14 Guler HP, Zapf J, Froesch ER. Short-term metabolic effects of recombinant human insulin-like growth factor I in healthy adults. N Engl J Med. 1987 Jul 16;317(3):137-40.

15 van der Klaauw AA, Biermasz NR, Feskens EJ, Bos MB, Smit JW, Roelfsema F, et al. The prevalence of the metabolic syndrome is increased in patients with GH deficiency, irrespective of long-term substitution with recombinant human GH. Eur J Endocrinol. 2007 Apr;156(4):455-62.

16 Handa Y, Maeda K, Toida M, Kitajima T, Ishimaru J, Nagai A, et al. Kabuki make-up syndrome (Niikawa-Kuroki syndrome) with cleft lip and palate. J Craniomaxillofac Surg. 1991 Apr;19(3):99-101.

17 Schott DA, Gerver WJM, Stumpel CTRM. Growth hormone therapy in children with Kabuki syndrome: 1-year treatment results. Horm Res Paediatr. 2017;88(3-4):258-64.

18 Schott DA, Gerver WJ, Stumpel CT. Growth hormone stimulation tests in children with Kabuki syndrome. Horm Res Paediatr. 2016; 86(5):319-24

19 Tanner JM. Growth at adolescence. 2nd ed. American Psychological Association; 1962.
20 Tanner JM, Whitehouse RH, Takaishi M. Standards from birth to maturity for height, weight, height velocity, and weight velocity: British children, 1965. II. Arch Dis Child. 1966 Dec;41(220):613-35.

21 Gerver WJM, de Bruin R. Paediatric morphometrics: a reference manual. Maastricht, Netherlands: Datawyse/Universitaire Pers Maastricht; 2001.

22 National High Blood Pressure Education Program Working Group on High Blood Pressure in $\mathrm{C}$, Adolescents. The fourth report on the diagnosis, evaluation, and treatment of high blood pressure in children and adolescents. Pediatrics. 2004 Aug;114(2 Suppl 4th Report):555-76.

23 Walter Greulich W, Idell Pyle S. Radiographic atlas of skeletal development of the hand and wrist. Stanford: Stanford University Press; 1959

24 Wallace TM, Levy JC, Matthews DR. Use and abuse of HOMA modeling. Diabetes Care. 2004 Jun;27(6): 1487-95.

25 Masson CJ, Mensink RP. Exchanging saturated fatty acids for (n-6) polyunsaturated fatty acids in a mixed meal may decrease postprandial lipemia and markers of inflammation and endothelial activity in overweight men. J Nutr. 2011 May;141(5):816-21.

26 Waterval WA, Scheijen JL, Ortmans-Ploemen MM, Habets-van der Poel CD, Bierau J. Quantitative UPLC-MS/MS analysis of underivatised amino acids in body fluids is a reliable tool for the diagnosis and followup of patients with inborn errors of metabolism. Clin Chim Acta. 2009 Sep;407(1-2): $36-42$.

27 Schott DA, Blok MJ, Gerver WJ, Devriendt K, Zimmermann LJ, Stumpel CT. Growth pattern in Kabuki syndrome with a KMT2D mutation. Am J Med Genet A. 2016 Dec;170(12): $3172-9$.

28 Lindgren AC, Hagenäs L, Müller J, Blichfeldt $\mathrm{S}$, Rosenborg M, Brismar T, et al. Effects of growth hormone treatment on growth and body composition in Prader-Willi syndrome: a preliminary report. The Swedish National Growth Hormone Advisory Group. Acta Paediatr Suppl. 1997 Nov;423:60-2. 
29 Bannink EM, van der Palen RL, Mulder PG, de Muinck Keizer-Schrama SM. Long-term follow-up of GH-treated girls with turner syndrome: metabolic consequences. Horm Res. 2009;71(6):343-9.

30 Oto Y, Tanaka Y, Abe Y, Obata K, Tsuchiya $\mathrm{T}$, Yoshino A, et al. Exacerbation of BMI after cessation of growth hormone therapy in patients with Prader-Willi syndrome. Am J Med Genet A. 2014 Mar;164A(3):671-5.

31 Rothermel J, Lass N, Bosse C, Reinehr T. Impact of discontinuation of growth hormone treatment on lipids and weight status in adolescents. J Pediatr Endocrinol Metab. 2017 Jul 26;30(7):749-57.

32 Martin DD, Wit JM, Hochberg Z, Sävendahl L, van Rijn RR, Fricke O, et al. The use of bone age in clinical practice - part 1. Horm Res Paediatr. 2011;76(1):1-9.

33 Cohen P, Bright GM, Rogol AD, Kappelgaard AM, Rosenfeld RG; American Norditropin Clinical Trials G. Effects of dose and gender on the growth and growth factor response to $\mathrm{GH}$ in GH-deficient children: implications for efficacy and safety. J Clin Endocrinol Metab. 2002 Jan;87(1):90-8.

34 Kang MJ, Kim EY, Shim YS, Jeong HR, Lee HJ, Yang S, et al. Factors affecting bone age maturation during 3 years of growth hormone treatment in patients with idiopathic growth hormone deficiency and idiopathic short stature: analysis of data from the LG growth study. Medicine. 2019 Apr;98(14) e14962.

35 Silha JV, Krsek M, Hana V, Marek J, Weiss V, Jezkova J, et al. The effects of growth hormone status on circulating levels of vascular growth factors. Clin Endocrinol. 2005 Jul;63(1):7986.
36 Kotler DP, Muurahainen N, Grunfeld C, Wanke C, Thompson M, Saag M, et al. Effects of growth hormone on visceral adipose tissue and dyslipidemia in HIV, an erratum. J Acquir Immune Defic Syndr. 2006 Nov 1;43(3): 378-80.

37 Zimmet P, Alberti KG, Kaufman F, Tajima N, Silink M, Arslanian S, et al. The metabolic syndrome in children and adolescents - an IDF consensus report. Pediatr Diabetes. 2007 Oct;8(5):299-306.

38 Giagulli VA, Castellana M, Perrone R, Guastamacchia E, Iacoviello M, Triggiani V. GH supplementation effects on cardiovascular risk in GH deficient adult patients: a systematic review and meta-analysis. Endocr Metab Immune Disord Drug Targets. 2017 Nov 16;17(4):285-96.

39 Ciresi A, Amato MC, Criscimanna A, Mattina A, Vetro C, Galluzzo A, et al. Metabolic parameters and adipokine profile during $\mathrm{GH}$ replacement therapy in children with GH deficiency. Eur J Endocrinol. 2007 Mar;156(3): 353-60.

40 Mogul HR, Lee PD, Whitman BY, Zipf WB, Frey M, Myers S, et al. Growth hormone treatment of adults with Prader-Willi syndrome and growth hormone deficiency improves lean body mass, fractional body fat, and serum triiodothyronine without glucose impairment: results from the United States multicenter trial. J Clin Endocrinol Metab. 2008 Apr;93(4):1238-45.

41 Rosen T, Bosaeus I, Tolli J, Lindstedt G, Bengtsson BA. Increased body fat mass and decreased extracellular fluid volume in adults with growth hormone deficiency. Clin Endocrinol. 1993 Jan;38(1):63-71.
42 de Boer H, Blok GJ, Voerman HJ, Phillips M, Schouten JA. Serum lipid levels in growth hormone-deficient men. Metabolism. 1994 Feb;43(2):199-203.

43 Juul A, Jørgensen JO, Christiansen JS, Müller J, Skakkeboek NE. Metabolic effects of GH: a rationale for continued $\mathrm{GH}$ treatment of $\mathrm{GH}$ deficient adults after cessation of linear growth. Horm Res. 1995;44 Suppl 3(Suppl 3): 64-72.

44 Meazza C, Elsedfy HH, Pagani S, Bozzola E, El Kholy M, Bozzola M. Metabolic parameters and adipokine profile in growth hormone deficient (GHD) children before and after 12-month GH treatment. Horm Metab Res. 2014 Mar;46(3):219-23.

45 Hoybye C. Inflammatory markers in adults with Prader-Willi syndrome before and during 12 months growth hormone treatment. Horm Res. 2006;66(1):27-32.

46 Andrade F, Llarena M, Lage S, Aldámiz-Echevarría L. Quantification of arginine and its methylated derivatives in healthy children by liquid chromatography-tandem mass spectrometry. J Chromatogr Sci. 2015 May-Jun; 53(5):787-92.

47 Gomez JM, Sahun M, Vila R, Domenech P, Catalina $\mathrm{P}$, Soler J, et al. Peripheral fibrinolytic markers, soluble adhesion molecules, inflammatory cytokines and endothelial function in hypopituitary adults with growth hormone deficiency. Clin Endocrinol. 2006 Jun; 64(6):632-9.

48 Topaloglu O, Sayki Arslan M, Turak O, Ginis Z, Sahin M, Cebeci M, et al. Three noninvasive methods in the evaluation of subclinical cardiovascular disease in patients with acromegaly: epicardial fat thickness, aortic stiffness and serum cell adhesion molecules. Clin Endocrinol. 2014 May;80(5):726-34. 